\title{
Acute urticaria with Angioedema in a patient with COVID-19 pneumonia: Favipiravir side effect or a rare cutaneous manifestation
}

Sevil Alkan Çeviker, Alper ŞENER, Cihan Yüksel, Taylan Önder, Anıl Akça, Servan Vurucu, Safiye Bilge Güçlü Kayta Çanakkale Onsekiz Mart Üniversitesi, Tıp Fakültesi, Enfeksiyon Hastalıkları ve Klinik Mikrobiyoloji Anabilim Dalı

\section{Abstract}

Introduction: Coronavirus disease 2019 (COVID-19) has caused thousands of deaths since it was declared as a pandemic. Recently it continues to be one of the most followed topics in the world in terms of its course and treatment.Favipiravir is a broad-spectrum anti-viral agent that has been shown to be effective against various Coronaviruses in vitro. However, as with any drug use, side effects may develop with the use of favipravir treatment.

Case Report: We reported a 55-year-old female patient with acute urticarial with angioedema whom had COVID-19 pneumonia. She had no history of allergy, atopy, previous similar episodes or family history of hereditary angioedema. There is no drug or food consumption that may be suspicious in terms of allergy described by the patient other than favipravir.
\end{abstract}

Conclusion: As far as we know, it is the first case reported from our country. Since there is no specific examination for differential diagnosis, we cannot distinguish as a rare side effect due to favipiravir treatment or COVID-19 cutaneous manifestation. As a result, studies involving more cases of COVID-19 skin findings are needed.

Keywords: COVID-19, Favipiravir, side effect, cutaneous manifestations, angioedema, acute urticaria.

\section{Introduction}

Coronavirus disease 2019 (COVID-19) has caused thousands of deaths since it was declared as a pandemic and continues to be one of the most followed topics in the world in terms of its course and treatment. ${ }^{1-3}$

Favipiravir, a new broad-spectrum antiviral drug developed years ago for influenza virus treatment, is a pyrazine carboxamide derivative that blocks replication by selectively inhibiting influenza viral RNA-dependent RNA polymerase. It is effective against many RNA viruses including H1N1, Ebola, Arena virus and Bunyavirus. ${ }^{1}$ Favipravir has been found to be effective in-vitro on Vero E6 cells infected with SARS-CoV-2 at high concentrations and shown in vitro against various coronaviruses.For these reasons it is one of the most widely used agents in the treatment of COVID-19 pneumonia in our country and over the world. ${ }^{2,3}$ However, as with any drug use, side effects may develop with the use of favipravir treatment. It is reported that the most common side effect in COVID-19 patients treated with favipiravir is diarrhea. Favipravir has been shown to moderately increase liver function tests even at therapeutic doses. ${ }^{4}$ However, sufficient literature information about skin side effects could not be reached.
We aimed to present a case of acute urticarial with angioedema who had COVID-19 pneumonia, that we can not distinguish as a rare side effect due to favipiravir treatment or COVID-19 cutaneous manifestation. As far as we know, it is the first case reported from our country.

\section{Case report}

A 55-year-old female patient,without a history of chronic disease, admitted to the pandemic outpatient clinic of our university hospital with tingling, swelling on the upper lip and emerging itchy maculopapular rashes on her trunk, face and abdomen for 8 hours duration (Picture 1).It was learned that, as her COVID-19 Real-time polymerase chain reaction (RT-PCR) test result was positive, favipravir treatment was started to her three days before. Except for the use of favipravir therapy, no drug, surgical, medical, and smoking history was reported.She had no history of allergy,atopy, previous similar episodes or family history of hereditary angioedema.She was not pregnant,too. There is no drug or food consumption that may be suspicious in terms of allergy described by the patient other than favipravir. Physical 


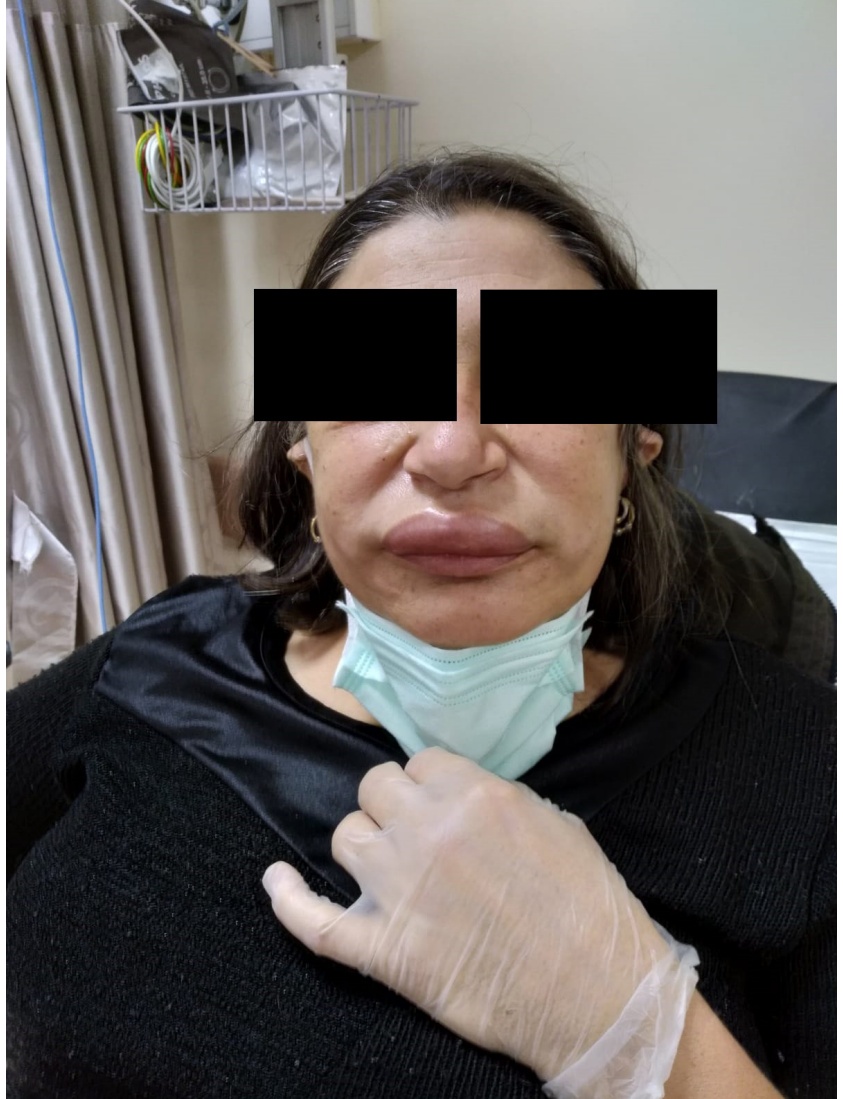

Picture 1

examination revealed that she had swollen lips and lower face, itchy maculopapular rashes on her trunk, the pharynx was oedematous (Picture 2) and mild crackles in the lung bases. Other system examinations were normal. She had no fever and cough. She had anosmia and ageusia. There was no periorbital or facial swelling. She was diagnosed as urticaria and angioedema afterdermatology consultation. The blood test results showed hemoglobin of $13.8 \mathrm{~g} / \mathrm{dl}$ (normal range adult females, $12-16 \mathrm{~g} / \mathrm{dl}$ ), white blood cell count of $12040 / \mathrm{ul}$ (normal range, 4000-10000/ul), platelets of 148000/ul (normal range, 165000-415000/ul), lymphocytes of 3180/ul (normal range, 800-2600/ul), C-reactive protein ( CRP) $1.301 \mathrm{mg} / \mathrm{dL}$ (normal range, $0-0,5$ ), blood urea of $18.8 \mathrm{mg} / \mathrm{dl}$ (normal range, $15-45 \mathrm{mg} / \mathrm{dl}$ ) and D-dimer of 128 (normal range, $<0.5$ ). Other investigations such as prothrombin time (PT), activated partial thromboplastin time (aPTT), international normalized ratio (INR) and liver enzymes were normal. No pathology was found in diagnostic tests for bacterial, viral and parasitic infections that could cause urticaria.Thorax tomography was compatible with viral pneumonia. The patient was hospitalized and hydroxychlorokin treatment $200 \mathrm{mg}$ 2X1 per oral to treat COVID-19 pneumonia and antihistaminic treatment was started with the recommendation of consultant physician. She received intravenous methylprednisolone, ranitidine, and levocetirizine dihydrochloride. A drug side effect notification was

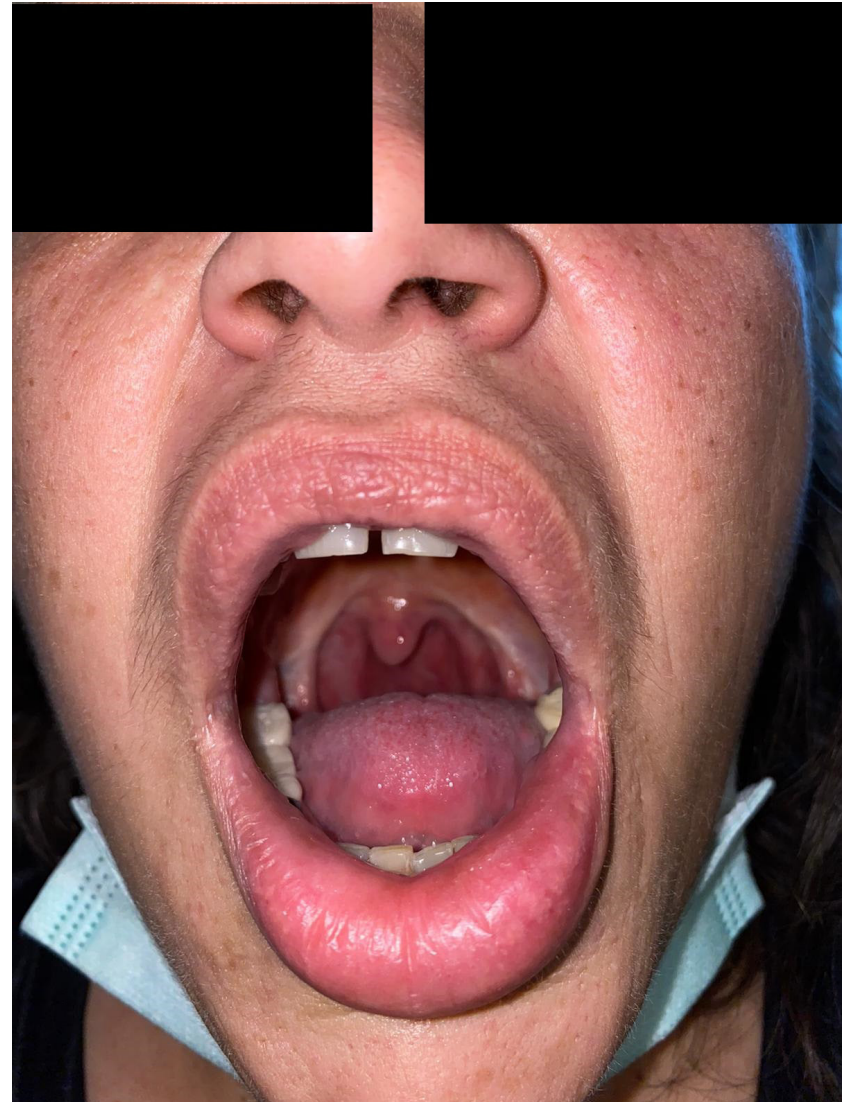

Picture 2

made to the Turkish Ministry of Health. By hospital day 2, the patient's complaints markedly improved. The patient, whose clinical findings were stable during the follow-up, had no dyspne and respiratory distress, was discharged.

\section{Discussion}

When the medical literature is examined, a variety of dermatologic eruptions thought to be related to COVID-19 has been reported, but angioedema has rarely been reported ${ }^{5-10}$. Najafzadeh et al. ${ }^{8}$ was reported similar case as and suggested that angioedemaas possible early diagnostic indicator of COVID-19 infection. However, while our patient had no complaints in the pre-treatment period, her complaints started on the third day of favipravir treatment.

The exact etiology of the skin findings associated with COVID-19 is not yet known. SARS-CoV-2 is thought to induce a mast cell activation, leading to histamine discharge..$^{5-8}$ Many hypotheses have been proposed in angioedema type 1 hypersensitivity reaction, often leading to an increase in vascular permeability or cross reaction between viral IgM and $\mathrm{IgG}$ promotes mast cell degranulation of mast cell Ig E or circulating immune complexes stimulate the production of vasoactive amines by basophils and activate com- 
plement. ${ }^{7-9}$ As general information, adverse drug reactions is divided into two groups asType A and Type B. Type A reactions are seen at a rate of $85-90 \%$, among all drug side effects. These reactions known usually dose-dependent. ${ }^{6-9}$ However, sufficient data on favipravir are not available yet. In the presented patient, urticaria and angioedema may be side effects due to favipravir or urticaria and angioedema due to histamine release. However, no differential diagnosis could be made. However, after favipravir was discontinued and the patient's antihistaminic treatment was arranged, the patient's complaints regressed.Urticaria and angioedema developed on the 3rd day of favipravir treatment in the patient, who had a diagnosis and symptoms of COVID-19 pneumonia before treatment but had no skin findings. Looking at the findings in the patient; both urticaria and angioedema were together, and the distinction between drug hypersensitivity reaction (Type 1 or 4) or COVID skin involvement could not be made clinically.

As seen in different case reports, COVID-19 pneumonia and urticaria/angioedema were seen in both genders. In the age group, it has been seen in patients from 30 to 60 years old. ${ }^{5-10}$ The patient we presented was a 55-year-old female patient.

In the study conducted by Recalcati ${ }^{10}$ in Italy, skin findings in 88 patients with coronavirus infection who did not take any new drugs within 15 days were evaluated. Skin findings developed in $18(20.4 \%)$ of these 88 patients; Erythematous rash in 14 patients $(16 \%)$, widespread urticaria in 3 patients $(3.4 \%)$ and varicella-like vesicles in 1 patient (1.1\%). ${ }^{4}$ However, it was reported that photographs were not taken due to the high risk of infection.

Usually mild lip involvement, periorbital, swelling of the eyelids and tongue were reported ${ }^{5-8}$ but in addition Hassan et al. ${ }^{5}$ had reported a case in which hand swelling was added. The patient we presented had swelling in the hands and legs and additional urticarial plaques in the trunk and retroauricular area.

\section{Conclusions}

As far as we know, it is the first case reported from our country. Since there is no specific examination for differential diagnosis, we cannot distinguish as a rare side effect due to favipiravir treatment or COVID-19 cutaneous manifesta- tion. As a result, studies involving more cases of COVID-19 skin findings are needed.

Conflicts of interest: The authors declare that they have no relevant conflicts of interest.

Informed consent: The patient gave written informed consent for publication (both clinical information and images).

\section{References}

1. Khambholja K, Asudani D. Potential repurposing of Favipiravir in COVID-19 outbreak based on current evidence. Travel Med Infect Dis. 2020;35:101710. doi: 10.1016/j. tmaid.2020.101710.

2. Cai $Q$, Yang $M$, et al. Experimental Treatment with Favipiravir for COVID-19: An Open-Label Control Study. Engineering (Beijing). 2020. doi: 10.1016/j. eng.2020.03.007

3. Galván Casas $C$, Català $A$, et al. Classification of the cutaneous manifestations of COVID-19: a rapid prospective nationwide consensus study in Spain with 375 cases. Br J Dermatol. 2020;183(1):71-77. doi: 10.1111/bjd.19163.

4. Marzano AV, Genovese $G$, et al. Varicella-like exanthem as a specific COVID-19-associated skin manifestation: multicenter case series of 22 patients. J Am AcadDermatol 2020;83(1):280-5. doi: 10.1016/j.jaad.2020.04.044.

5. Hassan K. Urticaria and angioedema as a prodromal cutaneous manifestation of SARS-CoV-2 (COVID-19) infection. BMJ Case Rep. 2020;13(7):e236981. doi: 10.1136/bcr-2020236981.

6. AbasaeedElhag $S A$, Ibrahim $H$, Abdelhadi S.Angioedema and urticaria in a COVID-19 patient: A case report and review of the literature. JAAD Case Rep. 2020;6(10):1091-1094. doi: 10.1016/j.jdcr.2020.07.042.

7. Adeliño R, Andrés-Cordón JF, Aracelis De La Cruz Martínez C. Acute urticaria with angioedema in the setting of coronavirus disease 2019. J Allergy Clin Immunol Pract. 2020;8(7):23862387. doi: 10.1016/j.jaip.2020.04.061.

8. Najafzadeh M, Shahzad F, Ghaderi N, Ansari K, Jacob B, Wright A. Urticaria (angioedema) and COVID-19 infection. J Eur Acad Dermatol Venereol. 2020;34(10):e568-e570. doi: 10.1111/jdv.16721.

9. Grewal E, Sutarjono B, Mohammed I. Angioedema, ACE inhibitor and COVID-19. BMJ Case Rep. 2020;13(9):e237888. doi: $10.1136 / \mathrm{bcr}-2020-237888$.

10. Recalcati S. Cutaneous manifestations in COVID-19: a first perspective. J Eur Acad Dermatol Venereol. 2020;34:e212e213 